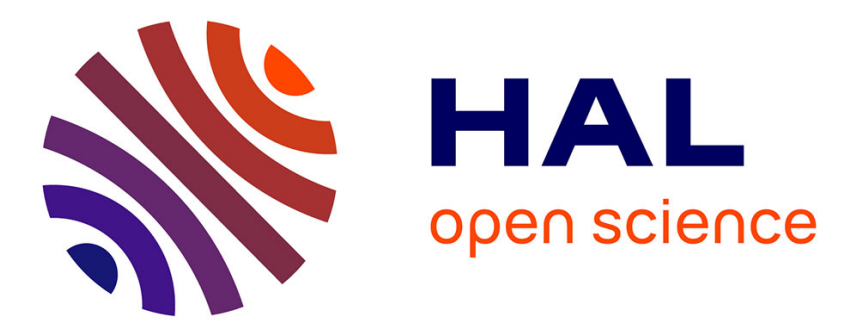

\title{
Dénonciation, régulation et réforme du droit de la famille par les groupes de pères séparés: ce que nous apprend la comparaison France-Québec
}

Aurélie Fillod-Chabaud

\section{- To cite this version:}

Aurélie Fillod-Chabaud. Dénonciation, régulation et réforme du droit de la famille par les groupes de pères séparés: ce que nous apprend la comparaison France-Québec . Revue canadienne femmes et droit, 2016, La régulation du genre dans la coexistence des niveaux de droit: regards croisés EuropeCanada, 28 (2), pp.617 - 645. hal-01547117

\section{HAL Id: hal-01547117 \\ https://hal-amu.archives-ouvertes.fr/hal-01547117}

Submitted on 8 Mar 2018

HAL is a multi-disciplinary open access archive for the deposit and dissemination of scientific research documents, whether they are published or not. The documents may come from teaching and research institutions in France or abroad, or from public or private research centers.
L'archive ouverte pluridisciplinaire HAL, est destinée au dépôt et à la diffusion de documents scientifiques de niveau recherche, publiés ou non, émanant des établissements d'enseignement et de recherche français ou étrangers, des laboratoires publics ou privés. 


\title{
Dénonciation, régulation et réforme du droit de la famille par les groupes de pères séparés : ce que nous apprend la comparaison France-Québec
}

\begin{abstract}
Aurélie Fillod-Chabaud
The recent mobilization of estranged fathers in France is the expression of a cause that emerged in the 1970s, following the feminist mobilizations of that time. In Europe, as in North America, these groups denounced a family justice that seemingly favours women by awarding them custody of the children and by relegating fathers to provider status, in terms of child-support payments. While these fathers hold egalitarian claims in promoting the sharing of a child's education after the parents' separation, their actions and discourses effectively support the traditional family unit by denouncing the "catastrophic" consequences of divorce on children. In light of a France-Québec comparison, we see different ways in which the regulation of conjugal separations is denounced by groups of estranged fathers.
\end{abstract}

Les récentes mobilisations de pères séparés en France sont l'expression d'une cause qui a émergé dans les années 1970, à la suite des mobilisations féministes de l'époque. En Europe, comme en Amérique du Nord, ces groupes dénoncent une justice familiale qui favoriserait les femmes en leur attribuant majoritairement la résidence des enfants et en ne reléguant les pères qu'à leur statut de pourvoyeur, par le paiement d'une pension alimentaire. Bien que ces pères portent des revendications égalitaires en faveur d'un partage éducatif des enfants à la suite de la séparation des parents, leurs actions et discours valorisent en fait le maintien de la cellule familiale traditionnelle, en dénonçant les conséquences « catastrophiques » du divorce sur les enfants. À la lumière de la comparaison France-Québec, nous verrons les différents aspects que prend la dénonciation de la régulation des séparations conjugales, par les groupes de pères séparés. 


\section{Table des matières}

1. La dénonciation de la régulation judiciaire des séparations conjugales : un processus généralisé depuis les années 1970

2. La perception du droit de la famille et de la régulation judiciaire par les groupes de pères séparés : une justice en faveur des femmes

2.1 Le sexe de la justice familiale

2.2 La féminisation " problématique » de la magistrature française

2.3 Assimiler la justice familiale à une pratique juridique peu prestigieuse

3. Le militantisme paternel : réformer la régulation judiciaire des séparations conjugales au-delà de l'échelle nationale

3.1 Des associations avant tout prestataires de services individuels

3.2 Une volonté politique similaire des deux côtés de l'Atlantique : sortir l'État de la régulation des séparations conjugales

Conclusion

Annexe 1

Annexe 2

«Benoît 2 ans sans papa : sauver nos enfants de la justice!». Ce slogan a été porté aux yeux des Français et des Françaises entre le 15 et le 18 février 2013 par Serge Charnay, un père divorcé, en haut d'une grue du chantier naval de Nantes ${ }^{1}$. Cette action, particulièrement bien diffusée par les médias, fut la première d'une longue série jusqu'en décembre de cette même année ${ }^{2}$. À chaque fois, un ou plusieurs pères se retranchaient pendant quelques heures en haut d'un monument pour

1. Serge Charnay, père de deux enfants, a un lourd dossier judiciaire, qui a dépassé les compétences de la justice civile. Entre autres, il n'a pas restitué son fils à sa mère à plusieurs reprises, à la fin de son droit de visite et d'hébergement. Voir par exemple : Oriane Dieulot, « Papa (séparé) est en haut [...] », L'Express (21 mars 2013) <www. lexpress.fr/actualite/societe/papa-separe-est-en-haut_1233229.html> ; « Nantes : Serge Charnay, retranché sur sa grue depuis 3 jours, va descendre ", L'Express et AFP (21 mars 2013) <www.lexpress.fr/actualite/societe/nantes-steve-charnay-retranche-sur-sagrue-depuis-3-jours-va-descendre_1221667.html> ; " Serge Charnay, l'homme de la grue nantaise, condamné à 4 mois de prison pour soustraction d'enfant », Le Huffington Post et AFP (11 mars 2014) <www.huffingtonpost.fr/2014/03/11/homme-grue-nantesserge-charnay-condamne-soustraction-fils_n_4942960.html>.

2. Une trentaine d'actions a été comptabilisée à partir d'articles de journaux, au cours de l'année 2013 dans différentes villes de France. Voir par exemple : en mai en haut de la Cathédrale d'Orléans ( "Droit de garde : huit pères en haut de la cathédrale d'Orléans », $L$ 'Express et $A F P(9$ mai 2013) <www.lexpress.fr/actualite/societe/droit-de-garde-huitperes-en-haut-de-la-cathedrale-d-orleans_1247523.html>), en juin en haut d'un pylône 
manifester un préjudice en lien avec la justice familiale et avec leur droit de garde. Ces actions ne sont pas les premières de la sorte : elles font écho aux interventions spectaculaires du groupe Fathers 4 Justice dans des pays anglo-saxons conduites depuis le début des années $2000^{3}$ et qui ont considérablement influencé les répertoires d'actions du militantisme paternel.

Bien qu'ostensiblement relayées depuis une quinzaine d'années, ces actions ne sont pas nouvelles. Les premiers mouvements d'hommes, dits mouvements « masculinistes », datent des années 1960-1970 et ont émergé en réponse à la deuxième vague du mouvement féministe. La théorie masculiniste remet en cause les rapports de domination hommes/femmes et s'inscrit dans une pensée « non-politiquement correcte » ayant pour principe de victimiser certaines classes dominantes de la société. Cette mouvance « englobe un ensemble d'individus et de groupes qui œuvrent à la fois pour contrer le féminisme et pour promouvoir le pouvoir des hommes $\gg{ }^{4}$. La rhétorique masculiniste est ainsi fondée sur un principe saillant : l'évolution de la condition féminine se ferait au détriment de celle des hommes, devenus objets de discriminations dans l'ensemble des secteurs de la société ${ }^{5}$. Au sein de la cellule familiale, le père aurait moins de légitimité à

dans les Côtes d'Armors ( « Trois pères perchés sur un pylône pour la garde de leurs enfants », Le Monde et AFP (28 juin 2013) <www.lemonde.fr/societe/article/2013/06/ 28/trois-peres-perches-sur-un-pylone-pour-la-garde-de-leurs-enfants_3438789_3224. html $>$ ) ou en octobre sur les toits de la place du Capitole à Toulouse (« Des pères en colère sur les toits de la place du Capitole », La Dépêche (2 octobre 2013) <www. ladepeche.fr/article/2013/10/02/1722129-peres-colere-toits-place-capitole.html>).

3. D'après la littérature sur les groupes de pères, la naissance en 2002 des Fathers 4 Justice en Angleterre fait concurrence à un des plus gros groupes anglo-saxons en direction des pères séparés, Families Need Fathers, fondé en 1974. Fathers 4 Justice s'impose alors par une nouvelle forme de militantisme plus radicale, visant à toucher l'opinion publique. Son fondateur Matt O'Connor dit faire preuve de désobéissance civile et initie une série d'actions qui ramènent leur nombre d'adhérents à 12000 en 2005. Ces actions ont également été adoptées par les F4J au Canada au milieu des années 2000, au cours d'une série de coups d'éclat en haut de monuments tels que le pont Jacques Cartier (Montréal), le pont Patulla (Vancouver) ou le City Hall (Toronto). Voir Richard Collier, "The Outlaw Fathers Fight Back: Fathers' Rights Groups, Fathers 4 Justice and the Politics of Family Law Reform: Reflections on the UK Experience » dans Richard Collier et Sally Sheldon, dir, Fathers' Rights Activism and Law Reform in Comparative Perspective, Oxford, Hart Publishing, 2008, 55 à la p 77 ; Robert A. Kenedy, Fathers For Justice: The Rise of a New Social Movement in Canada as a Case Study of Collective Identity Formation, Ann Arbor, Caravan Books, 2005.

4. Francis Dupuis-Déri et Mélissa Blais, dir, Le mouvement masculiniste au Québec : l'antiféminisme démasqué, Montréal, Remue-ménage, 2008 à la p 17.

5. Voir par ex Richard Collier et Sally Sheldon, « Fathers' Rights, Fatherhood and Law Reform: International Perspectives » dans Richard Collier et Sally Sheldon, dir, Fathers' Rights Activism and Law Reform in Comparative Perspective, Oxford, Hart Publishing, 2006, 1 aux pp 55-58. 
s'investir dans le travail parental ; au travail, les hommes subiraient des discriminations à l'embauche au nom de la parité et des quotas et une plus grande pénibilité dans l'exercice de leur profession; dans le monde politique ou encore au niveau de la santé enfin, ils seraient victimes des inégalités. C'est dans les années 1980 que le mouvement masculiniste va dénoncer les discriminations que les hommes subiraient devant la justice, notamment la justice familiale. Une partie du mouvement décide de cibler ses revendications sur les conséquences du divorce sur les enfants et sur la «disparition » des pères. Des associations de pères séparés émergent ainsi à cette période pour dénoncer les dysfonctionnements du traitement judiciaire des séparations conjugales qui favoriseraient l'absence des pères, et la multiplication des foyers monoparentaux, en fixant massivement la résidence des enfants chez leur mère et en poussant les pères à se désengager de leurs responsabilités éducatives pour ne rester que de simples pourvoyeurs, par l'intermédiaire de la pension alimentaire mensuelle.

C'est le caractère sexué du droit de la famille et du traitement judiciaire des séparations conjugales qui est dénoncé comme discriminant par le mouvement des pères $^{6}$ dans un contexte de massification des séparations conjugales dans tous les pays d'Europe et d'Amérique du Nord ${ }^{7}$. Au regard de la généralisation des répertoires d'actions de ces groupes qu'on observe des deux côtés de l'Atlantique, il est légitime de se demander s'ils formulent dans tous les pays des critiques similaires au sujet de la production et de l'application du droit familial auprès des pères séparés. En quoi ces revendications sont-elles liées aux systèmes et traditions judiciaires nationaux ? En quoi en sont-elles même la conséquence ?

On traitera de ces questions à la lumière d'une enquête réalisée dans le cadre d'une thèse de doctorat consacrée au militantisme paternel en France et au Québec entre 2008 et $2012^{8}$. Cette enquête a été conduite auprès d'associations de pères, d'institutions familiales et judiciaires et de représentants et représentantes politiques. La méthode ethnographique a été privilégiée : plus de 80 entretiens et une dizaine d'observations ont été réalisés. Un fichier de plus de 10000 adhérents d'une des plus importantes associations françaises a également été exploité statistiquement. Enfin, un travail d'analyse d'archives des diverses productions écrites des groupes (magazines, sites Internet, dépliants) a été réalisé.

6. Dans cet article, nous faisons le choix d'utiliser l'appellation de "mouvement » des pères pour souligner son caractère international, tandis que nous utiliserons le mot « groupe » lorsque nous parlerons d'une association spécifique, au niveau local ou national.

7. William Goode, World Changes in Divorce Patterns, Londres, Yale University Press, 1993 aux pp 25-26.

8. Aurélie Fillod-Chabaud, Au nom du père : Une sociologie comparative du militantisme paternel en France et au Québec, thèse de doctorat en sciences politiques et sociales, Institut Universitaire Européen, 2014 [non publiée] < doi.org/10.2870/589887>. 
La méthode comparative est un outil précieux pour analyser la manière dont les catégories du droit circulent et produisent des perceptions sexuées du judiciaire. En effet, la France et le Québec nous permettent d'inscrire cette comparaison dans l'analyse des similitudes (most similar systems design ${ }^{9}$ ), mais aussi des disparités (most different systems design ${ }^{10}$ ) de la divortialité et de son traitement judiciaire. Ces deux pays ont une tradition juridique francophone commune, par le biais du Code civil napoléonien, un fort taux de divortialité et à peu près les mêmes cadres de définition des modes de séparation ${ }^{11}$. Toutefois, les séparations ne sont pas régulées de la même façon : tandis qu'en France toutes les séparations de couples mariés sont régulées par la justice, le Québec est entré dans un processus de déjudiciarisation depuis une trentaine d'années ${ }^{12}$. Ces similitudes et différences produisent ainsi des ressentis communs et divergents sur la production et l'application du droit de la famille.

Dans cet article, nous assumons un certain déséquilibre dans le développement de nos études de cas : le cas français est davantage développé que le cas québécois qui a vocation à éclairer notre analyse et à affiner nos hypothèses de recherche. Nous supposons en effet que les revendications portées par le mouvement des pères reposent sur des piliers communs, quel que soit le pays au sein duquel il évolue. Toutefois, le traitement différencié des séparations conjugales et la politique de

9. Adam Przeworski et Henry Teune, The Logic of Comparative Social Inquiry, Michigan, Wiley-Interscience, 1970 à la p 37.

10. Theda Skocpol, States and Social Revolutions: A Comparative Analysis of France, Russia and China, Cambridge, Cambridge University Press, 1979 à la p 23.

11. Concernant la France, parmi les parents qui divorcent, les mères ont la résidence des enfants dans 70 pour cent des décisions, les résidences des enfants sont en alternance dans 21 pour cent des décisions, tandis qu'elle est fixée chez le père ou chez d'autres personnes respectivement dans 6 pour cent et 3 pour cent des décisions. Voir France, ministère de la Justice, Le regard des divorcés sur la résidence de leurs enfants, par Zakia Belmokhtar et Laurette Cretin, Infostat Justice 139, Paris, ministère de la Justice, (décembre 2012). Au Québec, la résidence est fixée chez la mère dans 60,5 pour cent des cas, elle est partagée dans presque 20 pour cent des cas et est confiée au père dans 13,5 pour cent des cas. Voir Émilie Biland et Gabrielle Schütz, La garde des enfants de parents séparés au Québec: Une analyse quantitative de dossiers judiciaires, Québec, Université Laval (L'ARUC), 2013 <www.fss.ulaval.ca/cms_recherche/upload/aruc_ famille/fichiers/que_savonsnous_5.pdf $>$.

12. En effet, sur le plan des séparations conjugales, plusieurs mesures ont été prises par le gouvernement québécois afin de sortir au maximum des murs du tribunal le règlement de la séparation. Ainsi, tout au long du processus judiciaire, la négociation est ouverte avec la partie adverse. Pour chaque procédure contentieuse, les justiciables sont poussés - par le biais de négociations entre les avocats ou avocates ou par la médiation - à ne pas mener leur procédure jusqu'au procès et donc à ne pas passer devant le tribunal. Source : Fondation du barreau du Québec, Seul devant la cour : En matières civiles, Québec, 2012. 
déjudiciarisation dans lequel le Québec s'est engagé depuis les années 1980, nous permettent de souligner une diversification du mouvement des pères québécois.

À ce titre, nous verrons tout d'abord qu'à l'échelle internationale, les groupes de pères séparés dénoncent communément la régulation judiciaire des séparations conjugales qui auraient des conséquences néfastes pour la société et pour les hommes en particulier (1). Ensuite, nous analyserons la perception du droit et de la régulation judiciaire par les groupes de pères (2). Enfin, l'outil comparatif nous permettra de saisir en quoi l'aspiration, certes marginale, de ces groupes à réformer le traitement judiciaire des séparations conjugales, défie les barrières des systèmes judiciaires nationaux (3).

\section{La dénonciation de la régulation judiciaire des séparations conjugales : un processus généralisé depuis les années 1970}

En Amérique du Nord, les groupes de défense des droits des pères apparaissent d'abord aux États-Unis, dans les années 1960, alors que les courbes de divorce sont en constante augmentation, mais connaissent leur véritable essor au début des années $1980^{13}$. Initialement, les groupes ciblent leurs revendications sur les conséquences financières du divorce et les enjeux liés à la pension alimentaire pour les enfants ou l'ex-conjointe, et non sur la garde et le droit de visite des enfants, comme ce sera le cas quelques d'années plus tard ${ }^{14}$. En 1965 est fondé le Committee for Fair Divorce and Alimony Laws, dont l'objectif principal est l'abolition de la pension alimentaire, alors que l'American Society for Divorced Men est créée en $1968^{15}$. Richard F. Doyle est celui qui parvient à regrouper les pères américains : il crée la Coalition of American Divorce Reform Elements

13. Jocelyn E Crowley, Defiant Dads: Fathers' Rights Activists in America, New York, Cornell University Press, 2008 aux pp 35-36 ; Anna Gavanas, Fatherhood Politics in the United States: Masculinity, Sexuality, Race and Marriage, Chicago, Illinois Press, 2004 aux pp 11-12, 22-23. Pour une vision globale de l'émergence des groupes de pères séparés, l'annexe 1 ci-dessous récapitule l'apparition des divers groupes de pères en France, au Québec et aux États-Unis des années 1960 aux années 2000. L'annexe 2 présente les principales caractéristiques des groupes rencontrés durant l'enquête doctorale et mobilisés dans cet article.

14. Le Men's Right Incorporated, un des rares groupes - marginaux à l'époque - axé sur la demande de garde n'est fondé qu'en 1970, dans le Massachusetts, par Fred Hayward. Voir Germain Dulac, Penser le masculin : essai sur la trajectoire des militants de la condition masculine et paternelle, Québec, Institut québécois de recherche sur la culture, 1994 à la p 57 [Dulac, Penser le masculin].

15. Ibid à la p 57 . 
(CADRE), en 1970, puis le Men's Rights Association (MRA), en $1973^{16}$. Le nombre de groupes de pères connaît une croissance considérable au cours de cette période : il passe de 185 en 1981 à plus de mille en $1985^{17}$.

Selon Jocelyn E. Crowley, sociologue du mouvement des pères aux États-Unis, la naissance en 1980 de la Joint Custody Association, issue d'une scission au sein du National Congress of Men, puis la création du National Congress for Fathers and Children dans les années 1990, annonce non seulement le glissement des regroupements d'hommes vers la cause paternelle, mais aussi celui des intérêts des pères vers la garde des enfants-les enjeux économiques du divorce étant plus secondaires $^{18}$. Ce processus est similaire au Canada ${ }^{19}$.

Les sociologues du militantisme paternel notent ainsi la diffusion de ce qu'ils appellent alors le Fathers' Rights Movement à partir des années 1990 dans plusieurs pays du monde, en Amérique du Nord, en Europe, mais aussi en Australie. Ces groupes ont en commun la volonté de réformer la justice familiale afin de mieux intégrer les pères dans l'éducation de leurs enfants à la suite de séparations conjugales. Ils se focalisent principalement sur les discriminations dont feraient l'objet les pères en cours de séparation ayant trait à la pension alimentaire, au droit de garde et au droit de la famille en général. À partir des années 2000, les groupes s'investissent davantage dans la réforme des politiques publiques et s'intègrent peu à peu dans le développement des antennes régionales ainsi que dans une démarche lobbyiste ${ }^{20}$.

L'analyse de la rhétorique de ces groupes fait ressortir une condamnation unanime des nouvelles configurations de la famille : le divorce serait néfaste à l'équilibre de la société, la monoparentalité conduirait à la délinquance infantile et l'absence des pères serait dévastatrice pour leurs enfants ${ }^{21}$. L'ensemble de la

16. Richard F Doyle expose sa vision de la régulation judiciaire du divorce dans son livre The Rape of the Male. Selon lui, les conditions sociales jouent en faveur des femmes et défavorisent les hommes au moment du divorce. La procédure du divorce favoriserait les femmes dans l'obtention de la garde des enfants et de la pension alimentaire, sans pour autant renforcer le droit de visite des pères. L'appareil juridique serait sexiste et favoriserait les mères dans l'attribution de la garde. Enfin, l'autorité paternelle doit demeurer au-delà de la séparation. Voir Dulac, Penser le masculin, supra note 14. Voir généralement Germain Dulac, «Le lobby des pères, divorce et paternité » (1989) 3:1 Revue Femmes et Droit 45.

17. Phyllis Chesler, Mothers on Trial: the Battle for Children and Custody, New York, McGraw Hill, 1986 à la p 426.

18. Crowley, supra note 13.

19. Dulac, Penser le masculin, supra note 14 à la p 56.

20. Gavanas, supra note 13.

21. Susan Boyd, " "Robbed of their families"? Fathers' Rights Discourses in Canadian Parenting Law Reform Processes » dans Richard Collier et Sally Sheldon, dir, Fathers' Rights Activism and Law Reform in Comparative Perspective, Oxford, Hart Publishing, 2008, 27 à la p 51 ; Carl Bertoia, "An Interpretative Analysis of the Mediation 
littérature sur le mouvement des pères montre qu'il a émergé autour de la promotion de la cellule familiale «traditionnelle » : le père et la mère sont la base de l'institution familiale et la remise en cause de la division des rôles genrés risque de la mettre en péril. Le premier numéro du magazine de Sos papa, publié en janvier 1991, donne le ton : «La structure familiale de la société française est bien en train de se désagréger [...] Nous pouvons nous demander ce qui participe à cette dégradation pour en interrompre le processus $»^{22}$. Le divorce et l'institution judiciaire sont très souvent considérés comme « la machine à séparer les familles », selon les groupes, qui s'inscrivent ainsi dans une véritable lutte contre le family breakdown ${ }^{23}$. Ils dénoncent par ailleurs la transmission et la reproduction de la dissociation familiale : les enfants de parents divorcés seraient condamnés à évoluer eux-mêmes dans une instabilité conjugale et à souffrir toute leur vie des conséquences de la séparation de leurs parents.

La moralisation des risques du divorce sur les enfants est un enjeu sans cesse mobilisé par les groupes de pères. La question des " enfants du divorce » et de la dissociation familiale a fait l'objet d'une vaste littérature présentant une vision moralisante et catastrophiste de ces nouvelles configurations familiales : le divorce symboliserait la fin de la famille nucléaire classique et le déclin de l'autorité paternelle ${ }^{24}$. Le magazine Sos papa illustre ainsi les conséquences « désastreuses » du divorce par de nombreux témoignages de jeunes adultes déchirés et traumatisés par la séparation de leurs parents. L'absence des pères est également dénoncée et imputée, non pas aux pères eux-mêmes, mais aux mères et aux institutions qui se substitueraient aux pères, notamment par les aides sociales. Une vaste littérature psychologique « pathologise » ainsi l'absence des pères et ses conséquences sur les enfants et, de la même façon, sur la société25. Plusieurs formes de déviances comme les troubles psychologiques, la « déviance sexuelle »-homosexualité des enfants_-ou encore les suicides sont ainsi assimilées à l'absence des pères, comme en témoigne cet extrait de prospectus :

Rhetoric of Fathers' Rights: Privatization versus Personalization » (1998) 16:1 Mediation Quarterly 15 ; Josianne Lavoie, « L'activisme juridique, le divorce et la garde des enfants : backlash sur les gains essentiels du mouvement féministe » dans DupuisDéri et Blais, supra note 4 à la p 179.

22. Michel Thizon, président de l'association Sos papa. Michel Thizon, "Prémonitoire et toujours d'actualité : La $1^{\circ}$ déclaration, fondatrice de 1'Association », Sos Papa (janvier 1991) <www.sos-papa.net/pages/declarationfondatrice.htm>.

23. Scott Coltrane et Neal Hickman, « The Rhetoric of Rights and Needs: Moral Discourse in the Reform of Child Custody and Child Support Laws » (1992) 39:4 Social Problems 400 à la $\mathrm{p} 413$.

24. Evelyne Sullerot, Le grand remue-ménage : La crise de la famille, Paris, Fayard, 1997.

25. Robert Bly, L'homme sauvage et l'enfant : l'avenir du genre masculin, Paris, Seuil, 1992 ; Guy Corneau, Père manquant, fils manqué, Montréal, Éditions de l'Homme, 1989. 
L'indifférence de la société à la souffrance psychique des enfants et des pères séparés entraîne des conséquences graves pour celle-ci. Sans père ni repère, ces enfants expriment souvent leur déséquilibre psychologique et affectif par l'échec scolaire, la drogue, parfois par le suicide et fréquemment par la délinquance. L'insécurité qui en résulte est ainsi directement générée par des acteurs sociaux et judiciaires eux-mêmes qui, de façon irresponsable, exercent encore une discrimination anti-père ${ }^{26}$.

Le slogan "manque de pères, manque de repères » est ainsi très régulièrement mobilisé par les groupes, qui n'hésitent pas à également se prononcer sur le caractère irremplaçable de la fonction paternelle, aussi bien au sein de débats sur les beaux-parents que sur l'homoparentalité ${ }^{27}$.

Par exemple, dans plusieurs lettres ouvertes adressées au gouvernement québécois, le président de l'Après-rupture ${ }^{28}$ enjoint les parlementaires à financer le groupe. Il légitime sa demande en avançant l'idée que ce financement participerait de manière indirecte à la lutte contre la délinquance juvénile : ces groupes permettraient de maintenir les liens pères-enfants après la séparation et de prévenir l'absence des pères dans l'éducation des enfants. Autre exemple, en France, alors que le président de la République Nicolas Sarkozy avait inscrit en 2007 la reconnaissance juridique du statut de beau-parent dans son agenda politique, la proposition de loi formulée par la secrétaire à la Famille, Nadine Morano, est abandonnée le 31 mars 2009 sous la pression des groupes de pères ${ }^{29}$. Naviguant entre parenté sociale et biologique, les membres de Sos Papa se saisissent de ce débat, craignant de se faire remplacer par les beaux-pères de leurs enfants. " Avant de donner davantage de droits aux beaux-parents, donnons-en aux pères!" "clament-ils en réunions $s^{30}$.

L'actualité de ces débats sur les conséquences des dissociations familiales et de l'absence des pères prend d'autant plus de relief que la France et le Québec doivent

26. Prospectus de Sos papa : voir SOS Papa, « 2.000.000 d'enfants privés de père ! » $<$ www.sos-papa.net/pages/tract.htm>.

27. Bly, supra note 25 ; Corneau, supra note 25.

28. Le groupe l'Après-rupture est présenté dans l'annexe 2, ci-dessous.

29. Voir par exemple, Joël Plantet, « Un statut pour le beau-parent ? », Lien Social (22 novembre 2007) <www.lien-social.com/Un-statut-pour-le-beau-parent> ; « SOS Papa se prononce contre le statut du beau-parent ", L'Obs (3 août 2007) <tempsreel. nouvelobs.com/societe/20070803.OBS9261/sos-papa-se-prononce-contre-le-statut-dubeau-parent.html > AFP, « Le « statut du beau-parent » loin de faire l'unanimité », 20 minutes (3 août 2007) <www.20minutes.fr/france/173539-20070803-statut-beauparent-loin-faire-lunanimite>.

30. Cette citation a été relevée par l'auteure dans son carnet de terrain à l'automne 2008 . 
faire face à une judiciarisation massive des séparations conjugales ${ }^{31}$. Ainsi depuis la fin des années 1990, l'institution judiciaire est au cœur des critiques des groupes de pères, cette dernière produisant, selon eux, une justice en faveur des femmes.

\section{La perception du droit de la famille et de la régulation judiciaire par les groupes de pères séparés : une justice en faveur des femmes}

Attardons-nous à présent sur les principales attaques formulées par le mouvement des pères français en direction de la justice familiale. Tout d'abord, celle relative au sexe du juge et à son influence sur la prise de décision (2.1). Ensuite, celle portant sur la féminisation jugée " problématique » des professions judiciaires engagées dans les affaires familiales (2.2), avec, en creux, la critique d'une pratique peu prestigieuse du droit (2.3).

\subsection{Le sexe de la justice familiale}

« Vous êtes jugés à Toulouse ? Vous avez de la chance, ce tribunal est plutôt propères »; " vous passez devant cette juge ? Préparez-vous, elle en fait baver aux hommes et elle est contre la résidence alternée pour les enfants de moins de 6 ans. " Ces avertissements et injonctions sont tout à fait courants parmi les habitués et les nouveaux venus dans les réunions de groupe auxquelles nous avons assisté : tous y vont de leurs conseils et de leurs remarques sur les pratiques judiciaires de tel ou tel juge ou tel ou tel tribunal. En effet, les groupes de pères n'ont de cesse de dire que l'attribution massive de la résidence des enfants chez la mère est due à une complicité entre la justice familiale et les mères rendant inaudibles les demandes des pères. Cette complicité serait, en France, une des conséquences de la forte

31. En 2008, l'indicateur conjoncturel de divortialité (cet indicateur permet d'estimer le nombre de divorce dans une promotion fictive de 100 mariages dont les taux de divorce seraient à chaque durée de mariage égaux à ceux observés l'année considérée) est de 45 pour cent en France. L'indice synthétique de divortialité de 30 ans pour 100 mariages (correspond à la proportion de couples mariés qui, en principe, divorceront avant leur $30^{\mathrm{e}}$ anniversaire) est de 40,7 pour cent en 2008 au Canada, et de 47,4 pour cent au Québec, la même année. Voir l'Institut national d'études démographiques (INED), Divorces <www.ined.fr/fr/tout-savoir-population/chiffres/france/mariagesdivorces-pacs/divorces $>$; Statistique Canada, Les causes de divorce traitées par les tribunaux civils en 2010-2011, par Mary Bess Kelly, dans Juristat, no de catalogue 85-002-X, Ottawa, Statistique Canada, $2012<$ www.statcan.gc.ca/pub/85-002-x/2012001/ article/11634-fra.htm>. 
féminisation de la magistrature et serait le fait au Québec d'un féminisme d'État ${ }^{32}$ qui toucherait tous les corps de l'administration.

Les recherches sur la justice familiale permettent de nuancer ce constat. Le groupe de recherche Ruptures ${ }^{33}$ sur le traitement judiciaire des séparations conjugales (auquel j'appartiens), sur la base d'observations d'audiences de séparations conjugales et de statistiques produites à partir de dossiers archivés de séparations, montre par exemple que la résidence des enfants est un sujet peu débattu lorsque les parents se rendent au tribunal (le premier conflit est en lien avec la pension alimentaire). Ce sont principalement les mères qui héritent de la résidence de leurs enfants et les pères sont nombreux à ne pas exprimer ni de désaccord voire d'opinion sur ce sujet ${ }^{34}$.

Quant à l'incidence du sexe du magistrat ou de la magistrate sur le jugement, on observe en fait deux situations différentes en France et au Québec. Tout d'abord, les accusations de surféminisation avancées par les groupes de pères sont peu mobilisées au Québec car le corps de la magistrature y est bien moins féminisé qu'en France ${ }^{35}$. De même, aucune étude n'a à ce jour testé l'incidence du sexe sur les jugements aux affaires familiales au Québec, il est donc impossible de savoir si

32. Selon Anne Revillard, le féminisme d'État (state feminism) renvoie d'une façon générale à la présence féministe dans l'État et aux actions étatiques en faveur des femmes. Voir Anne Revillard, La cause des femmes dans l'État. Une comparaison FranceQuébec, Grenoble, Presses Universitaires de Grenoble, 2016, à la p 18.

33. Ce groupe de recherche veut contribuer à la connaissance des justices familiales dans les deux pays. Adossé à des enquêtes de terrain réalisées dans chacune des nations selon un protocole voisin, il vise à construire une sociologie comparée du traitement judiciaire des séparations conjugales en France et au Québec. En France comme au Québec, cette recherche souhaite enrichir l'expertise scientifique en sociologie du droit et de la famille. Présentation de la recherche sur le site suivant : voir Projet Ruptures $<$ www.ruptures.ulaval.ca $>$.

34. Voir Céline Bessière, Émilie Biland et Aurélie Fillod-Chabaud, « Résidence alternée : la justice familiale face aux rapports sociaux de sexe et de classe » (2013) 69 Lien social et Politiques 125 aux pp 130-32<www.erudit.org/revue/lsp/2013/v/n69/ 1016488ar.pdf > ; Biland et Schütz, supra note 11 ; Collectif Onze, Au tribunal des couples : Enquête sur des affaires familiales, Paris, Odile Jacob, 2013 ; Renée Joyal, "L'enfant dont la garde est contestée : sa place dans le processus de décision » (1996) 37:1 Cahiers de Droit 51 à la p 59 <www.erudit.org/revue/cd/1996/v37/n1/ 043378ar.pdf $>$.

35. En 2013, selon une base de données produite par l'équipe québécoise du groupe de recherche « Ruptures », parmi l'ensemble des juges de la Cour supérieure du Québec, 32 pour cent sont des femmes. Voir Émilie Biland et Gabrielle Schütz, « Tels pères, telles mères? La production des déviances parentales par la justice familiale québécoise » (2014) 97:4 Genèses à la p 46. 
un tel biais existe ${ }^{36}$. En France, la magistrature est quant à elle en voie de féminisation et les affaires familiales attirent de nombreuses magistrates. Le Collectif Onze s'est ainsi interrogé sur les éventuelles incidences du sexe des juges aux affaires familiales (JAF) dans les décisions judiciaires, à la demande notamment de nombreuses professionnelles de la justice, inquiètes de rendre des jugements partiaux au regard de la polémique déclenchée par les groupes de pères depuis quelques années $^{37}$. À ce propos, l'enquête montre que les jugements rendus par les juges hommes et femmes des quatre juridictions étudiées sont d'une grande homogénéité :

Parmi les 276 dossiers concernant des enfants dans notre base de données, dans $73 \%$ des cas leur résidence habituelle est fixée chez la mère, dans $10 \%$ des cas chez le père, dans $13 \%$ c'est une résidence alternée et dans $4 \%$ la résidence des différents enfants n'est pas fixée chez le même parent. [C]ette répartition ne varie que très peu selon le sexe du juge [...] la structure des décisions - fixation massive de la résidence chez la mère, et de façon minoritaire fixation d'une résidence alternée ou chez le père-est identique dans les deux cas, avec $72 \%$ des décisions pour les magistrates femmes, et plus de trois quarts des décisions pour les magistrats hommes fixant la résidence chez la mère ${ }^{38}$.

Si l'étude insiste sur des « styles » de juge différents, selon leur sexe, mais aussi selon leur trajectoire professionnelle, les jugements rendus varient peu. En outre, lorsque les parents judiciarisent leur séparation, seuls 10 pour cent des affaires présentent un désaccord sur la résidence des enfants ${ }^{39}$. En cas de désaccord sur la résidence, le ou la juge fixe deux fois plus de résidence chez le père que dans les situations d'accord entre parents.

Les associations de pères ont toutefois l'habileté de médiatiser des statistiques sur la résidence des enfants sans préciser si elles sont issues d'un accord des parents

36. Le courant de recherches gender and judging est toutefois florissant en Amérique du Nord et de nombreuses études ont proposé des analyses genrées des jugements à la fois quantitativement (différences des décisions prises entre hommes et femmes) et qualitativement (la manière dont les juges rendent leurs décisions et leurs raisons) (voir par exemple Dermot Feenan, «Women and Judging » (2009) 17:1 Feminist Legal Studies 1 aux pp 4-7) mais aucune étude ne s'est spécifiquement intéressée au cas des affaires familiales au Québec.

37. Collectif Onze, supra note 34.

38. Ibid aux pp 157-58.

39. Résultats issus de la dernière étude du ministère de la Justice sur la résidence des enfants selon les demandes des parents. L'étude porte sur 6042 décisions définitives. Voir France, ministère de la Justice, La résidence des enfants de parents séparés : De la demande des parents à la décision du juge. Exploitation des décisions définitives rendues par les juges aux affaires familiales au cours de la période comprise entre le 4 juin et le 15 juin 2012, par Maud Guillonneau et Caroline Moreau, Paris, ministère de la Justice, novembre $2013<$ www.justice.gouv.fr/art_pix/1_rapportresidence_11_2013.pdf >. 
ou non, afin de mieux servir leur cause et de perpétuer la tradition d'une remise en cause de la féminisation des professions de prestige au sein notamment de la haute fonction publique ${ }^{40}$. Médias et hautes instances de contrôle prêtent ainsi une oreille attentive aux « dérives » occasionnées par la forte féminisation de cette profession, alors que bon nombre de corps professionnels, les métiers de la politique par exemple, ne sont pas encore à la parité. Cette féminisation est ainsi unanimement considérée comme « problématique ${ }^{41}$.

\subsection{La féminisation " problématique » de la magistrature française}

En France, la féminisation de la magistrature génère depuis ces vingt dernières années une série de fantasmes autour des pratiques judiciaires, comme l'a montré la sociologue du droit Anne Boigeol ${ }^{42}$. Cette féminisation a en effet suscité des résistances au milieu du $20^{\mathrm{e}}$ siècle, les membres des jurys de l'École nationale de la magistrature (ENM) émettant notamment des réserves sur les capacités des femmes à juger. Lorsque cette féminisation s'est avérée inévitable, dans les années 1970 , ces jurys ont commencé à redouter une féminisation « excessive » de ce corps de profession ${ }^{43}$. Marlaine Cacouault-Bitaud montre également combien les magistrates sont soupçonnées depuis des décennies d'exercer leur profession avec empathie $^{44}$ : les hommes incarneraient une justice asexuée et impartiale ; les femmes seraient renvoyées à leur propre nature et leur propre sensibilité ${ }^{45}$.

La dénonciation de pratiques judiciaires en faveur des femmes par les groupes de pères relance ainsi le débat sur la féminisation de la magistrature, comme en témoigne notamment le discours de Christiane Taubira, à l'époque garde des Sceaux, à la promotion 2012 de l'ENM en décembre de cette année-là, en appelant

40. À titre d'exemple, les groupes utilisent les statistiques sur la fixation de la résidence des enfants (environ 70 pour cent des résidences sont fixées chez la mère) en détournant les chiffres de la manière suivante : «70\% des enfants coupés de leurs pères ! »; «70\% des pères considérés comme des mauvais pères par la justice familiale ! » etc.

41. Voir par exemple le discours de Christiane Taubira, infra note 46.

42. Anne Boigeol, « Les femmes et les Cours. La difficile mise en œuvre de l'égalité des sexes dans l'accès à la magistrature » (1996) 22 Genèses 116.

43. Ibid à la p 121 .

44. Marlaine Cacouault-Bitaud, « La féminisation d'une profession signifie-t-elle une baisse de prestige ? » (2001) 5:1 Travail, genre et sociétés 93 aux pp 104-07, 111 $<$ doi.org/10.3917/tgs.005.0091>.

45. Juliette Rennes, Le mérite et la nature, une controverse républicaine : L'accès des femmes aux professions de prestige (1880-1940), Paris, Fayard, 2007. Voir notamment la seconde partie de l'ouvrage, chapitre 1. 
de ses vœux la réintroduction des hommes parmi les professions judiciaires ${ }^{46}$. Le secteur des affaires familiales est d'autant plus investi par des juges femmes que les hommes avouent fuir au plus vite cette fonction considérée par eux comme intrusive et très peu juridique ${ }^{47}$. Les femmes-jeunes pour la plupart- $\mathrm{y}$ voient quant à elles une possibilité de concilier leur vie de famille avec leurs charges professionnelles, cette fonction leur permettant notamment de rédiger les jugements à leur domicile et d'avoir des horaires « de bureau $»^{48}$.

Cette féminisation de la profession de juges aux affaires familiales (JAF) n'échappe pas aux pères membres de la cause paternelle. Elle sert notamment d'argument au moment de l'affaire de la grue à Nantes_brièvement évoquée en introduction-pour le président de Sos papa, Fabrice Méjias, dénonçant la forte attribution de la résidence des enfants chez leur mère : "La justice est sexiste. Sinon comment expliquer ces disparités ? La loi est en théorie la même pour tous. Elle n'est pas et ne doit pas être appliquée en fonction du genre. Le sexisme, la déviance de nos institutions et de notre droit naissent lors de jugements où père et mère ne sont clairement pas traités sur un pied d'égalité. ${ }^{49}$ Cette mise en cause est perceptible depuis la création de Sos papa, au sein des magazines de l'association. À titre d'exemple, dans un numéro de décembre 1993, les auteurs dénoncent la vision, selon eux stéréotypée, que les JAF se font du père et de la mère : l'un est représenté par un homme de Cro-Magnon, bourru en non civilisé ; l'autre est féminine, contemporaine et rassurante. La publication d'une affiche en 2013 par le 《Printemps des pères $»^{50}$, dénonçant les pratiques pro-femmes des JAF et assimilant les initiales de cette fonction à celle d'une "Justice affligeante féminine ",

46. «Progressivement nous aurons à faire en sorte qu'il y ait plus d'hommes dans les prochaines promotions, mais je pense que les garçons vont revenir assez vite ». Voir AFP, « Taubira: il faut attirer davantage vers la magistrature, les hommes notamment », Le Parisien (17 décembre 2012) <www.leparisien.fr/bordeaux-33000/taubira-il-fautattirer-davantage-vers-la-magistrature-les-hommes-notamment-17-12-2012-

2415157.php>.

47. Le sexe des juges aux affaires familiales en poste n'est à ce jour pas communiqué par la Chancellerie. À tout le moins, en 2011, 68 pour cent des juges non spécialisés des tribunaux de grande instance sont des femmes. Au sein de l'enquête du Collectif Onze, sur les 28 juges aux affaires familiales en poste, 20 sont des femmes. Collectif Onze, supra note 34 à la p 125.

48. Voir Collectif Onze, supra note 34 au chapitre 3 ; Céline Bessière et Muriel Mille, «Le juge est (souvent) une femme. Conceptions du métier et pratiques des magistrates et magistrats aux Affaires familiales » (2013) 55:3 Sociologie du travail 341 à la p 356.

49. Renée Greusard, "Grues occupées : « Merci aux deux pères nantais » », Rue 89 (16 février 2013) <rue89.nouvelobs.com/2013/02/16/grues-occupees-merci-aux-deux-peresnantais-pour-cette-action-239699>.

50. Dans le cadre des actions menées par les pères français depuis février 2013, une manifestation est organisée le 21 mars 2013, appelée "Printemps de pères ", dans plusieurs villes de France. 
nous montre que cette problématique est toujours d'actualité. La très grande proportion de femmes au sein de l'institution judiciaire familiale n'a ainsi cessé depuis des décennies d'être considérée comme problématique par les groupes de pères et d'être l'objet d'une vaste entreprise de décrédibilisation, à laquelle prennent part les professionnels du droit, partisans de la cause des pères.

\subsection{Assimiler la justice familiale à une pratique juridique peu prestigieuse}

En creux, c'est une pratique peu prestigieuse du droit qui est dénoncée, comme le montre cet extrait d'entretien avec un avocat ${ }^{51}$ de Sos papa en 2008 :

Pour résumer la situation je pourrais dire, en caricaturant un petit peu c'est vrai, mais à peine et avec une finalité pédagogique, je pourrais dire que encore aujourd'hui dans ce monde judiciaire du juge aux affaires familiales qui est quasiment exclusivement féminin, mais ça c'est un problème dans le problème, encore aujourd'hui quotidiennement, y a une [...] une perception de l'homme comme étant dangereux, comme étant un prédateur, et une perception de la femme comme étant, par essence une victime devant être protégée [...] Donc non seulement la magistrature est ultraféminisée, alors les avocats sont en passe de le devenir, on est à peu près à $50 / 50$, mais avec 51 pour les femmes et 49 pour les hommes, ils sont en train de basculer. Et néanmoins, dans les affaires familiales, c'est frappant y a que des femmes magistrates, que des femmes avocates. Pratiquement [...] Donc si vous voulez tout ça accentue le fait que dans la pratique judiciaire, et là je parle d'une pratique judiciaire au quotidien, il n'y a que des femmes. Et c'est vrai que l'univers étant uniquement matriarcal et féminin et ben, on se demande ce qu'on fait là, les hommes se demandent ce qu'ils font là. On a presque envie de s'excuser.

L'avocat reprend ici la vision de la justice défendue par Sos papa et réitère la critique d'une trop forte féminisation comme étant à l'origine de décisions en faveur des mères et à l'encontre des pères. Cette féminisation est associée à une baisse de prestige - la profession ayant perdu son monopole masculin. Comme les enseignants et les enseignantes du secondaire sont confrontés à des classes de plus en plus nombreuses, les juges aux affaires familiales sont de plus en plus confrontés à une justice de masse, ils et elles ont alors « [p] eu d'occasions de briller par des

51. Cet avocat de Sos papa est un des six avocats qui font du conseil juridique à l'association auprès des pères en ruptures conjugales. Dans le cadre de ma thèse, j'ai eu l'occasion de réaliser un entretien avec lui à son cabinet à Paris, en mai 2008. Voir Fillod-Chabaud, supra note 8 aux pp 309-12. 
constructions juridiques toutes en finesse [...] où il faut parfois sacrifier la qualité au rendement. L'autorité du juge se trouve quelque peu banalisée dans ce type de justice $\aleph^{52}$. Cette justice de l'urgence serait ainsi le signe d'une baisse de prestige et attirerait de fait moins de candidats masculins ${ }^{53}$.

Cette dévalorisation est d'autant plus forte que les métiers ayant trait à la famille, aux enfants et au travail social sont extrêmement féminisés. Selon ce raisonnement, les magistrates et les avocates sont accusées de manque d'objectivité face à l'évidence de la «neutralité masculine ${ }^{54}$. Ce manque de neutralité et d'objectivité supposé chez les JAF femmes les pousserait à motiver leur décision, non pas en fonction du droit, mais en fonction de leur ressenti marqué par l'affect ou l'empathie, les femmes étant toujours suspectées d'incompétence technique, parce que ce sont des femmes, comme l'explique encore ici cette avocate de Sos papa:

Alors parmi les magistrats il y a, je dirais l'immense majorité, qui n'ont pas de démarche je dirais « volontairement discriminatoire » envers les hommes je tiens à le préciser. Y a quelques magistrates qui restent tout à fait connues par les avocats, par exemple en Île-de-France [...] qui sont ouvertement je dirais même pas pro-mère, mais anti-hommes [...] Dans l'immense majorité des cas ça n'est pas ça, je veux dire qu'il n'y a pas de discours militant ni d'attitude anti-homme ou anti-masculine [...] donc c'est plus [...] c'est pas du militantisme. Mais en même temps, je sais pas si c'est plus ou moins grave, mais c'est préoccupant parce que tout simplement une femme qu'elle soit magistrate ou pas, elle est de son temps, elle est de son ère et elle est imprégnée, comme un avocat, de son socioculturel, si vous voulez y a un moment, en dépit de votre formation, de votre pratique professionnelle, vous êtes avant tout ce que vous êtes. Ok ? Donc vous transportez avec vous vos préjugés et votre mode de pensée à un instant donné. Et, moi je trouve [...] y a si vous voulez y a un savoir judiciaire et une pratique professionnelle évidemment qui est éthique, tout est scrupuleusement motivé dans les décisions de justice, le travail est bien fait, mais le problème dans les affaires familiales, c'est que c'est pas un raisonnement de droit. Vous avez une décision humaine qui est habillée et justifiée par du droit, mais il faut être très clair, si y a un endroit dans la justice,

52. Anne Boigeol, « Les magistrates de l'ordre judiciaire : des femmes d'autorité » (1997) 97:1 Les Cahiers du Mage 25 à la p 27 <recherche.parisdescartes.fr/mage/content/ download/1208/5150/version/1/file>.

53. Il s'agit en effet d'une justice de masse et de l'urgence. Les chambres aux affaires familiales en France traitent chaque année selon le ministère de la Justice 400000 nouvelles procédures, ce qui représente la moitié du contentieux civil des tribunaux de grande instance. Par ailleurs, la cadence judiciaire est très soutenue : selon le Collectif Onze, une audience dure en moyenne 18 minutes et un ou une juge traite 800 affaires par an. Voir Collectif Onze, supra note 34 à la p 15 .

54. Rennes, supra note 45. 
dans la machine judiciaire où on juge d'abord avec ses tripes, si je peux m'exprimer ainsi, et qu'ensuite on habille, parce qu'il faut bien, d'articles de loi, c'est les affaires familiales ${ }^{55}$.

Dans cet extrait, le droit de la famille est assimilé à une pratique peu noble, parce qu'il ne nécessiterait pas une pratique du droit élaborée : selon l'avocate, il s'agit davantage de « juger avec ses tripes » qu'avec ses acquis juridiques. Comme le souligne M. Cacouault-Bitaud, le fait de juger « avec ses tripes » pour les femmes suppose qu'elles le font avec émotion et un sentiment de solidarité féminine. Ici les usages militants du droit se font par la dévalorisation d'une profession jugée peu légitime au sein du champ juridique par un de ses pairs.

Ainsi, nous avons montré combien la justice familiale est considérée par les groupes de pères comme une institution plus proche des intérêts féminins que masculins, justement par sa forte féminisation. Cette forte féminisation donne lieu à une critique de fond sur le fonctionnement et les pratiques de cette institution. Par ailleurs, la critique interne de l'avocat de Sos papa à l'encontre des magistrates est le signe d'une concurrence au sein du champ judiciaire : les avocats représentent une profession libérale et critiquent la haute fonction publique, schéma assez commun dans les luttes entre corps professionnels.

Le cas français nous permet ainsi de saisir la nature de la critique des groupes de pères à l'égard de la justice familiale et plus spécifiquement à l'égard des femmes juges. Quelles solutions sont proposées par les groupes pour remédier à ce qu'ils considèrent comme une justice en faveur des femmes ? Encore une fois, la comparaison nous éclaire sur ce point : si seulement quelques groupes portent, en France comme au Québec, une réforme du traitement des séparations conjugales, il s'avère qu'elle prend la même forme, dans des contextes nationaux différents.

\section{Le militantisme paternel : réformer la régulation judiciaire des séparations conjugales au-delà de l'échelle nationale}

Les groupes rencontrés ${ }^{56}$ en France et au Québec se différencient par leur mode de fonctionnement, les origines de leurs financements et la composition de l'équipe qui anime les activités en direction des pères séparés. Dans ces deux pays, les groupes prennent soit une forme « associative militante », c'est-à-dire qu'ils sont composés de bénévoles et ont été créés sous l'impulsion d'un ou deux individus, soit une forme " institutionnelle », c'est-à-dire qu'ils sont composés de professionnels (travailleurs sociaux, psychologues) et ont été créés sous l'impulsion d'une institution (service d'action sociale d'une commune, etc.) ${ }^{57}$. Par « ressources

55. Voir Fillod-Chabaud, supra note 8 à la p 311.

56. Pour rappel, les associations rencontrées sont présentées en annexe 2 ci-dessous.

57. Voir annexe 2 ci-dessous. 
institutionnelles ", nous entendons cependant que ces ressources peuvent concrètement prendre la forme d'une association, ou d'un organisme communautaire au Québec. En France, les dispositifs en direction des pères séparés prennent principalement la forme de ressources associatives militantes et exceptionnellement celle de ressources institutionnelles ${ }^{58}$. À titre d'exemple, les cinq associations rencontrées sont des ressources associatives militantes : dans notre panel, nous n'avons pas de ressources institutionnelles, même si nous avons appris, au cours de l'enquête, leur existence marginale ${ }^{59}$. Au Québec, le paysage du militantisme paternel est en voie de diversification : les ressources institutionnelles deviennent de plus en plus nombreuses et ce dans une volonté de contrer une vision de la paternité revendicative et violente. Ces ressources promeuvent une vision pacifiée et déjudiciarisée de la séparation, en écho à la politique de déjudiciarisation engagée par le Québec il y a une trentaine d'années ${ }^{60}$. Ainsi, seules trois des neuf associations de pères rencontrées correspondent à ce que nous appelons des ressources associatives militantes ${ }^{61}$.

À la lumière de cette typologie, à la suite des rencontres que nous avons eues avec ces groupes, nous présenterons les différentes aspirations des groupes, selon leur nature : si tous sont prestataires de services individuels (3.1), seules les ressources militantes promeuvent une réforme de la régulation des séparations conjugales, qui est similaire, malgré leurs systèmes judiciaires différents (3.2).

\subsection{Des associations avant tout prestataires de services individuels}

Trois types de services sont proposés par les groupes rencontrés en France et au Québec : un service d'accueil et d'échange, un service d'aide psychologique, et du conseil juridique ${ }^{62}$. L'aide juridique est le domaine le plus développé, de deux

58. Voir annexe 2 ci-dessous.

59. Le psychologue français Emmanuel Gratton fait part de l'existence de groupes institutionnels dans le cadre d'une étude d'un groupe de pères animé par un travailleur social et un thérapeute familial dans l'enceinte d'une Caisse d'allocations familiales dans l'Ouest de la France. L'écoute et la parole y sont plus valorisées que le conseil juridique. Voir Emmanuel Gratton, «Un groupe de parole de pères divorcés et séparés. Entre égalité parentale et solidarité masculine » (2012) 9:1 Recherches familiales 173 à la p $186<$ doi.org/10.3917/rf.009.0173>.

60. Pour plus de détails sur les usages différenciés du droit par le mouvement des pères en France et au Québec, voir Aurélie Fillod-Chabaud, "Les usages du droit par le mouvement des pères séparés : Une comparaison France-Québec » (2016) 15:1 Genre sexualité \& société $<$ gss.revues.org/3746>.

61. Voir annexe 2 ci-dessous.

62. Voir annexe 2 ci-dessous. 
façons distinctes. Dans les ressources associatives militantes, le suivi est généralement assuré par des non-professionnels.

Il s'agit de pères séparés socialisés au droit familial au moment de leur rupture et qui conseillent les pères sur les différentes démarches à adopter. Il est possible dans un deuxième temps, dans certaines de ces structures, de s'adresser à un avocat mis à disposition par le groupe, mais cela engendre des coûts. Les avocats bénévoles (qui font des consultations juridiques à Sos papa, par exemple) acceptent en entretien individuel de donner des conseils " généraux », mais refusent de donner des conseils personnalisés et proposent, à défaut, d'être en charge de leur dossier, en tant qu'avocat rémunéré. Le suivi juridique individualisé est assuré par des professionnels (juristes, avocats) dans les ressources institutionnelles. Salariés ou bénévoles, ils sont obligés de respecter une charte « éthique » et ne peuvent " débaucher » les pères venus les voir pour devenir leur avocat. Il s'agit d'ailleurs souvent d'avocats à la retraite.

Seules les ressources associatives militantes formulent une opposition aux pouvoirs publics. Elles critiquent tout d'abord la manière dont la justice traite les séparations conjugales et portent un projet de déjudiciarisation par la promotion, notamment, de la médiation familiale. Ce projet est principalement porté par la $F M C P$ : le groupe propose de remplacer l'ordonnance de conciliation (ONC) première étape judiciarisée d'une procédure de divorce — par une séance de médiation familiale gratuite dans une structure de proximité. La médiation deviendrait ainsi un passage obligatoire, relativement similaire au modèle québécois, par lequel tous les parents se séparant devraient passer ${ }^{63}$.

Un groupe français et un groupe québécois portent tous les deux le projet de supprimer les prestations compensatoires, pour la France, et la pension alimentaire sans terme pour conjoint, pour le Québec. L'Association des nouvelles conjointes du Québec (ANCQ) ou Sos divorce en France plaident, d'une part, pour une prise en charge par l'État de la compensation financière de la dissolution conjugale et pour l'intégration des femmes sur le marché du travail et, d'autre part, pour une limitation des pensions alimentaires ou prestations compensatoires. Ainsi l'ANCQ prône une série de réformes qui mettraient fin aux prélèvements d'argent des hommes vers leurs ex-conjointes, les femmes divorcées étant particulièrement

63. La médiation familiale française s'est en effet largement inspirée du Québec. Alors que la reconnaissance de la médiation familiale par la Loi sur le divorce date au Canada de 1985 (art 8), la constitution du Comité national des associations et services de médiation familiale en France en 1991 se fait en référence directe à l'exemple québécois. Voir Revillard, supra note 32 à la p 122. 
bien protégées par l'État, selon sa présidente, Lise Bilodeau ${ }^{64}$. De la même manière, lors de la réforme de la prestation compensatoire en France en 2000, Sos divorce avait demandé et avait obtenu à défaut de la suppression totale de la prestation compensatoire (leur demande originelle), la suppression de la rente viagère.

Enfin, la cause la plus unanimement portée par les groupes de pères, français comme québécois, est celle de la résidence alternée «par défaut ». La présomption de garde partagée au Québec, appelée loi C-22 puis C-422, fait écho à un ensemble de projets de loi portés par des députés français de différents bords politiques depuis 2009, dont nous allons voir à présent l'historicité et la portée politique.

\subsection{Une volonté politique similaire des deux côtés de l'Atlantique : sortir l'État de la régulation des séparations conjugales}

Les critiques formulées par les groupes de pères à l'encontre de la justice vont de pair avec une série de demandes de réformes du droit de la famille, en France et au Québec. Ce sont principalement des projets de réforme qui promeuvent la résidence alternée (dite « garde partagée » au Québec) «par défaut »: il s'agit d'imposer ce mode de garde si au moins un des deux parents le demande. Le but de ce projet est de contrer les mères qui refuseraient systématiquement un mode de garde plus égalitaire et d'empêcher le ou la juge d'intervenir dans cette décision. La présomption de résidence alternée servirait justement à limiter le pouvoir discrétionnaire du ou de la juge lié à la notion d'intérêt de l'enfant, présente dans les deux Codes civils nationaux ${ }^{65}$. En 1998, une réflexion est lancée sur la présomption de la garde partagée au Canada, dans le cadre de la réforme de la Loi sur le divorce ${ }^{66}$. Un rapport, intitulé Pour l'amour de nos enfants, est produit dans ce but, par un comité mixte du Sénat à la suite d'une consultation publique auprès de groupes de pères ${ }^{67}$. Malgré la promotion de la garde partagée dans l'ensemble

64. L'Association des Nouvelles Conjointes du Québec (ANCQ) est un groupe fondé par Lise Bilodeau qui se soucie aussi bien de la discrimination subie par les hommes au cours de leur divorce/séparation que de la situation des nouvelles conjointes qui doivent être solidaires financièrement des dépenses que leurs conjoints doivent engager pour leurs ex-épouses (pension alimentaire). L'association milite également pour la présomption de la garde partagée. Voir ANCQ, "Historique »<http://ancq.qc.ca/ notre-association/historique-2>.

65. Le Québec est la seule province canadienne à être dotée d'un Code civil. Le Québec régit le droit de la famille par le biais du Code civil, mais il est également soumis à la tradition jurisprudentielle émanant de la common law nord-américaine.

66. Loi sur le divorce, LRC 1985, c 3 ( $2^{\mathrm{e}}$ supp).

67. Canada, Sénat et Chambre des communes, Comité mixte spécial sur la garde et le droit de visite des enfants, Rapport du comité mixte spécial sur la garde et le droit de visite

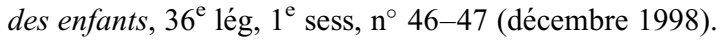


du rapport, une conclusion modérée est au final proposée, préconisant que la garde partagée ne peut être imposée par défaut, toutes les familles n'ayant pas les mêmes besoins et les mêmes demandes. Le projet de loi C-22 est finalement « mort au feuilleton » (abandonné) en 2002. À cette époque, des groupes québécois comme l'ANCQ ou l'Après-rupture se sont engagés pour plaider en faveur de la loi C-22, alors que le Québec et l'Alberta sont dans des dispositions juridiques différentes du reste du Canada. En effet, à ce jour, les provinces canadiennes-à l'exception de ces deux provinces - ne dissocient pas l'autorité parentale (guardianship) de la résidence physique de l'enfant (le parent qui n'a pas la résidence de son enfant n'a pas le même droit de regard sur les décisions importantes qui le concernent), alors que l'autorité parentale commune est indépendante de la résidence de l'enfant en France et au Québec depuis une vingtaine d'années ${ }^{68}$.

Le débat du C-22 est alors relancé en 2011 par des députés de l'Ouest canadien au travers du nouveau projet de loi C-42269. Le projet est mis au feuilleton quelques semaines plus tard. Malgré les va-et-vient du texte dans le débat politique, deux fonctionnaires du ministère de la Justice, rencontrés en juin 2011, ne croient pas en l'aboutissement du projet :

Enquêtrice : Qu'est-ce que ça aurait comme conséquence pour le Québec si la loi C-422 passait?

Fonctionnaire 1 : Nous on va considérer que ça va à l'encontre de l'intérêt de l'enfant, on va s'opposer. C'est certain, d'ailleurs les ministres de la Justice de toutes les provinces ont indiqué au ministre de la Justice du Canada qu'ils étaient contre. [...] Si jamais ça passe ça s'appliquerait en matière de divorce seulement, et la moitié du volume judiciaire des dossiers à la Cour supérieure c'est des dossiers de divorce, qui diminuent de plus en plus. Ça veut dire que la moitié des gens qui se séparent seraient assujettis à cette condition. Ça risque d'envenimer les conflits, ça peut faire en sorte qu'on ait un taux de contestation qui est pas de $10 \%$ là, qui va peut-être augmenter. C'est ça que ça donnerait : une augmentation de la judiciarisation de la contestation.

Fonctionnaire 2 : Mais ça fait déjà plusieurs années qu'il y a des motions comme ça.

Fonctionnaire $1:[\ldots]$ Et que ça passe jamais parce que c'est présenté par un député et non par un ministre donc on le laisse mourir au feuilleton, ce dossier-là.

Le fait que ces juristes ne croient aucunement en l'adoption de cette loi au Québec est une des raisons de ses abandons et propositions successives, non par des ministres, mais par des députés. Une loi orientant à ce point la pratique des juges ne serait, en effet, pas envisageable.

68. Art 605-606 CcQ ; art 373-2 C civ (France).

69. PL C-422, Loi modifiant la Loi sur le divorce, $2^{\mathrm{e}}$ sess, $40^{\mathrm{e}}$ lég, 2009. 
En France, de la même manière qu'au Québec, et dans un cas de figure où l'autorité parentale ${ }^{70}$ est également dissociée de la résidence des enfants, la présomption de résidence alternée est sur la scène politique depuis 2009, comme nous allons le voir dès à présent. Cette question connaît toutefois une actualité politique virulente à la fin de l'année 2013 en lien notamment avec les mobilisations de pères tout au long de cette année-là71.

La loi du 4 mars 2002 (légalisation de la résidence alternée dans le Code civil) a été considérée comme l'aboutissement d'années de lutte au sein de l'association Sos papa. La hausse relative du taux de résidence alternée depuis le début des années 2000 serait toutefois le signe, non d'une faible demande des parties selon l'association, mais d'une pratique judiciaire inchangée ${ }^{72}$. Selon Sos papa, les juges auraient du mal à accorder ce mode de résidence, surtout auprès d'enfants en bas âge. De la même manière, les avocats pousseraient les pères à ne pas formuler une demande de résidence alternée par peur d'être déboutés. Ainsi, depuis 2009, l'association s'est lancée, en partenariat avec des députés de droite (UMP), dans le façonnement d'une proposition de loi sur la résidence alternée comme mode de résidence " par défaut » sur le même principe que le projet canadien. Une première proposition de loi est formulée le 18 mars 2009 par un groupe de parlementaires sous la direction des députés Richard Maillié et Jean-Pierre Decool ${ }^{73}$. Rédigée de manière grossière, à l'aide de chiffres hasardeux et d'affirmations psychologisantes, cette proposition fait peu parler d'elle aussi bien dans les médias que dans l'opinion publique. Un an et demi plus tard, la proposition de loi est réactualisée et suivie d'une publication dans le journal Le Monde, en faveur de la résidence alternée par une quinzaine de personnalités - dont une seule femme-aussi bien politiciens, psychologues, sociologues que professionnels de la santé. Plusieurs mois après cette publication, et après l'alternance présidentielle de mai 2012, une autre proposition de loi est soumise par le député Jean-Pierre Decool et d'autres parlementaires en faveur de la résidence alternée par défaut ${ }^{74}$. Cette proposition est quasi similaire

70. En France, l'autorité parentale reste exercée conjointement dans 98 pour cent des divorces. Voir France, ministère de la Justice, L'exercice de l'autorité parentale après le divorce ou la séparation des parents non mariés, par Laure Chaussebourg et Dominique Baux, Paris, 2007.

71. Voir supra notes 1 et 2.

72. De 2003 à 2012, les procédures de divorce aboutissant à une résidence alternée ont augmenté de 9 points, passant de 12 à 21 pour cent. Voir France, ministère de la Justice, Les décisions des juges concernant les enfants de parents séparés ont fortement évolué dans les années 2000, par Valérie Carrasco et Clément Dufour, Infostat Justice 132, Paris, Ministère de la justice, janvier $2015<$ www.justice.gouv.fr/art_pix/ stat_Infostat $\% 20132 \% 20$ def.pdf $>$.

73. France, Assemblée nationale, « Proposition de loi $n^{\circ} 1531$ visant à privilégier la résidence alternée pour l'enfant dont les parents sont séparés » (18 mars 2009) <www.assembleenationale.fr/13/pdf/propositions/pion1531.pdf >.

74. France, Assemblée nationale, « Proposition de loi $n^{\circ} 1531$ visant à préserver l'autorité partagée et à privilégier la résidence alternée pour l'enfant en cas de séparation des parents » (24 octobre 2012) <www.assemblee-nationale.fr/14/pdf/propositions/ pion0309.pdf>. 
à la précédente et, bien que relativement relayée dans les médias, reste encore une fois lettre morte.

L'année 2013 marque toutefois un tournant dans la politisation de la cause paternelle. En effet, dès février 2013, des pères interpellent les pouvoirs publics sur la résidence alternée. La rencontre entre la ministre de la Justice et la ministre déléguée à la Famille, quelques jours après la première action de Serge Charnay en haut d'une grue nantaise, est suivie d'une consultation sur le respect de la coparentalité entre les parents séparés. Commandé le 7 juin 2013, ce rapport, rendu au début de l'année 2014, est composé d'un ensemble de réflexions sur la parentalité post-conjugale issues de plusieurs séances réunissant des groupes et institutions en lien avec la famille ${ }^{75}$. En parallèle de cette consultation, un autre événement relance le débat sur la résidence alternée par défaut, à l'automne 2013. Dans le cadre des débats qui se sont tenus au Sénat sur la loi pour l'égalité des hommes et des femmes, un amendement a été voté dans la nuit du 17 au 18 septembre 2013 pour favoriser la résidence alternée par défaut. Le contenu de cet amendement est le suivant : il s'agit d'imposer aux JAF de motiver la non mise en place d'une résidence alternée lorsqu'un des deux parents la demande ${ }^{76}$. Contrairement à ce que l'on pourrait croire au vu de l'historique présenté en amont, il s'agit d'un amendement proposé par Françoise Laborde, membre du Rassemblement démocratique et social, courant majoritaire du Parti radical de gauche. Lorsqu'elle le présente, son argumentaire s'inscrit dans un héritage plutôt féministe (elle cite notamment Françoise Héritier) sur l'égalité professionnelle entre hommes et femmes et la nécessité de changer les pratiques à la fois au travail et à la maison. Elle suggère la résidence alternée par défaut parmi d'autres propositions afin de créer un changement des pratiques éducatives et domestiques - et professionnelles-en légiférant sur la nécessité pour les pères de s'investir dans l'éducation des enfants avant et après la séparation. Cet amendement voté au milieu de la nuit est immédiatement critiqué par la ministre des Droits des femmes de l'époque, Najat VallaudBelkacem, alors que des journalistes rédigent des articles stipulant que le Sénat défend les droits des pères ${ }^{77}$.

75. France, Groupe de travail coparentalité DACS-DGCS, Rapport sur les réflexions $d u$ groupe de travail sur la coparentalité : "Comment assurer le respect de la coparentalité entre parents séparés ", Paris, Ministère de la justice et Ministère des affaires sociales et de la santé, janvier 2014.

76. France, JO, Sénat, Débats parlementaires, Compte rendu intégral, séance du 17 septembre $2013<$ www.senat.fr/seances/s201309/s20130917/s20130917023.html>.

77. Voir notamment les articles suivants : Delphine Bivona, «Le Sénat souhaite rendre prioritaire la résidence alternée : comment se prévaloir de cette avancée ? ", Village de la Justice (12 mars 2014) <http://www.village-justice.com/articles/Senat-souhaiterendre-prioritaire,16413.html> ; Gaëlle Dupont, « Le Sénat encourage la garde alternée, contre l'avis du gouvernement ", Le Monde (18 septembre 2013) <www.lemonde.fr/ societe/article/2013/09/18/le-senat-encourage-la-garde-alternee-contre-1-avis-dugouvernement_3479705_3224.html>. 
Le 24 janvier 2014, lors de la discussion des articles du projet de loi à l'Assemblée nationale, N. Vallaud-Belkacem s'oppose formellement à l'introduction d'un tel amendement sur la résidence alternée par défaut et ce d'autant plus que ce sujet lui semble éloigné dudit projet de loi. La ministre soulève l'aspect problématique de légiférer sur des dispositions dont il revient au tribunal d'évaluer leur adéquation avec la situation du couple et de l'enfant. Ensuite, une réflexion sur la coparentalité est mise en place par la ministre déléguée Dominique Bertinotti dans le cadre d'un projet de loi sur la famille ${ }^{78}$. Cette proposition de loi (dite « loi Famille : autorité parentale et intérêt de l'enfant ») est remise à l'agenda politique par la nouvelle secrétaire d'État en mai 2014, Laurence Rossignol. La résidence alternée par défaut ne figure toutefois pas dans ce projet de loi, les conclusions du rapport sur la coparentalité déconseillant la mise en place d'une telle présomption. Au moment de la rédaction de cet article, la proposition de loi n'a pas évolué depuis son adoption en première lecture par l'Assemblée nationale, en juin 2014.

Des deux côtés de l'Atlantique, comment expliquer un tel échec des projets de réformes sur la présomption de résidence alternée ? Cet échec est à mettre en lien avec la nature même de la réforme proposée par les groupes de pères séparés : la présomption de résidence alternée est inconcevable pour les parlementaires car elle suppose un contrôle du pouvoir discrétionnaire du tribunal. En cas de désaccord sur la résidence, il semble difficile d'inscrire dans le marbre une situation par défaut. À ce titre, il est intéressant de noter que la principale demande des groupes en matière de réforme familiale vise justement cet aspect de la pratique judiciaire car c'est précisément à la part discrétionnaire de la nature féminine dans le processus décisionnel que les groupes cherchent à mettre fin. Le cas du Québec est un peu différent : si les juges femmes ne sont pas directement à l'origine de l'attribution massive de la résidence des enfants chez leurs mères, le fait de pouvoir imposer une pratique par défaut lorsqu'un des deux parents souhaite une résidence alternée permettrait, selon les groupes, d'inverser durablement cette tendance.

\section{Conclusion}

Cet article s'est centré sur les critiques formulées par les groupes de pères séparés sur la régulation judiciaire des séparations conjugales. L'outil comparatif a permis d'isoler les incidences des traditions et régulations judiciaires propres à chaque pays dans la nature des critiques formulées par les groupes. Tout d'abord, la dénonciation du traitement judiciaire des séparations conjugales est un processus commun aux groupes depuis les années 1970, et ce essentiellement parce que ces

78. Ce projet est reporté sine die, alors qu'il devait être examiné à la mi-février 2014, sous la pression des associations familiales contre l'adoption et la procréation médicalement assistée par les couples homosexuels et notamment l'association La manif pour tous, devenue célèbre lors des débats sur la loi du mariage pour tous en 2013. 
séparations seraient néfastes à la stabilité de la société toute entière. Le mouvement des pères émerge en effet autour de la dénonciation des conséquences du divorce sur la cellule familiale en général et le lien père-enfant en particulier. Encore aujourd'hui, les revendications de ce mouvement gravitent autour de l'irremplaçabilité de la fonction paternelle et la valorisation de la paternité biologique. Ensuite, le cas français nous a permis d'analyser les critiques formulées à l'égard de la justice familiale par les groupes de pères, à la lumière de la structure du corps professionnel judiciaire. Cette critique est centrée sur la féminisation de la profession de JAF et la manière dont ces femmes juges produisent des décisions judiciaires qui seraient " anti-pères ». En effet, les groupes de pères médiatisent l'attribution massive de la résidence des enfants chez leur mère - attribution qui n'est pourtant pas liée à des décisions arbitraires de la part des juges aux affaires familiales-et mettent en lien ces chiffres avec la forte féminisation de la magistrature, féminisation qui serait le signe d'une pratique du droit partiale et peu rigoureuse. Enfin, des deux côtés de l'Atlantique, la typologie des groupes de pères nous permet de mieux contextualiser l'émergence des groupes dans des situations de régulations judiciaires différentes : nous y apprenons que tous les groupes ne s'engagent pas dans une réforme du droit, certains ne ciblant leurs actions que sur la prestation de services auprès de pères séparés. La présence massive de ressources institutionnelles au Québec et leur promotion commune de la sortie du processus judiciaire est ainsi à mettre en lien avec les politiques de déjudiciarisation engagées depuis plusieurs années. Il semble toutefois que, dans le cadre de revendications de réformes législatives sur la résidence des enfants, la tradition juridique pèse peu sur la nature des réformes demandées : les groupes français comme québécois font la promotion commune de la régulation de la pratique judiciaire des juges dans l'espoir de mettre fin à ce qu'ils considèrent comme une justice en faveur des femmes. 
Annexe 1 : Émergence du mouvement des pères divorcés aux États-Unis, au Québec et en France : une chronologie récapitulative constituée à partir de la littérature sur les mobilisations de pères séparés

\begin{tabular}{|c|c|c|c|c|}
\hline Nom & Année & Par & Devient/ Scission & En \\
\hline \multicolumn{5}{|l|}{ Etats-Unis } \\
\hline Free Men Inc. & 1960 & & $\begin{array}{l}\text { Quatre universitaires du Maryland } \\
\text { Devient Coalition of Free Men }\end{array}$ & 1980 \\
\hline Committee for Fair Divorce and Alimony & 1965 & Sidney Siller & & \\
\hline American Society for Divorced Men & 1968 & Charles Metz & & \\
\hline Men's Right Incorporated & 1970 & Fred Hayward & & \\
\hline $\begin{array}{l}\text { Coalition of American Divorce Reform } \\
\text { Elements (CADRE) }\end{array}$ & 1970 & Richard F. Doyle & Devient Men's Rights Association & 1973 \\
\hline Men's Rights Association & 1973 & Richard F. Doyle & Devient Men International & 1980 \\
\hline National Congress for Men & 1980 & Joseph A. Barbier & $\begin{array}{l}\text { Scission et naissance de la } \\
\text { Joint Custody Association }\end{array}$ & 1980 \\
\hline National Congress for Fathers and Children & 1990 & & & \\
\hline Promise Keepers & 1990 & Bill McCourtney & & \\
\hline \multicolumn{5}{|l|}{ Québec } \\
\hline Hom-Info & 1980 & & Des universitaires de Montréal & \\
\hline $\begin{array}{l}\text { Association des Hommes Séparés et Divorcés } \\
\text { de Montréal }\end{array}$ & 1983 & & $\begin{array}{l}\text { Groupe d'entraide aux pères et de } \\
\text { soutien à l'enfant }\end{array}$ & 1987 \\
\hline L'Autonhommie & 1984 & & & \\
\hline \multicolumn{5}{|l|}{ Fathers for Equality in Divorce } \\
\hline \multicolumn{5}{|l|}{ Montréal Men's Network } \\
\hline Réseau Homme Québec & 1992 & Guy Corneau & & \\
\hline $\begin{array}{l}\text { Regroupement pour la Valorisation de la } \\
\text { Paternité }\end{array}$ & 1996 & Yvon Lemay et Manuel Prats & & \\
\hline \multicolumn{5}{|l|}{ Repère } \\
\hline \multicolumn{5}{|l|}{ Orpères } \\
\hline L'Après-rupture & 1996 & Gilbert Claes & & \\
\hline
\end{tabular}


Annexe 1: suite

\begin{tabular}{|c|c|c|c|c|}
\hline Nom & Année & Par & Devient/ Scission & En \\
\hline Pères Séparés Inc. & 1997 & & & \\
\hline Fathers 4 justice & 2003 & Benoît Leroux & & \\
\hline \multicolumn{5}{|l|}{ France } \\
\hline $\begin{array}{l}\text { Défense des intérêts des divorcés hommes et } \\
\text { de leurs enfants mineurs (Didhem) }\end{array}$ & 1975 & Marc Droulez & $\begin{array}{l}\text { Devient le Mouvement pour la } \\
\text { Condition Masculine et Paternelle } \\
\text { (Puis scission en } 2 \text { groupes) : }\end{array}$ & 1975 \\
\hline Mouvement pour la Condition Masculine & 1975 & Antoine Leenhardt & Devient Sos Divorce & 2000 \\
\hline Mouvement pour la Condition Paternelle & 1977 & Paul Elkaim & $\begin{array}{l}\text { Scission en } 2 \text { mouvements : } \\
\text { le } M E P \text { et la } F M C P \text { (voir ci-dessous) }\end{array}$ & \\
\hline Mouvement pour l'Egalité Parentale & 1980 & Paul Elkaim & & \\
\hline $\begin{array}{l}\text { Fédération des Mouvement pour la Condition } \\
\quad \text { Paternelle }\end{array}$ & 1984 & Stéphane Ditchev & & \\
\hline Sos Papa & 1990 & Michel Thizon & & \\
\hline Les Papas $=$ Les Mamans & 2006 & Gérard Révérend & & \\
\hline
\end{tabular}


Annexe 2 : Principales caractéristiques des groupes rencontrés durant l'enquête doctorale

\begin{tabular}{|c|c|c|c|c|c|c|c|}
\hline Nom & But & $\begin{array}{l}\text { Date } \\
\text { création }\end{array}$ & Ressource & $\begin{array}{l}\text { Services } \\
\text { proposés }\end{array}$ & & & $\begin{array}{l}\text { Changements } \\
\text { législatifs proposés }\end{array}$ \\
\hline FMCP & $\begin{array}{l}\text { Promotion de la } \\
\text { médiation familiale } \\
\text { et des droits des } \\
\text { pères }\end{array}$ & 1974 & $\begin{array}{l}\text { Associative } \\
\text { militante }\end{array}$ & $\begin{array}{l}\text { Des animateurs } \\
\text { accueillent et con- } \\
\text { seillent personnelle- } \\
\text { ment les adhérents }\end{array}$ & Lieu de réflexion & $\begin{array}{l}\text { Lieu d'action auprès } \\
\text { des institutions et } \\
\text { des professionnels }\end{array}$ & $\begin{array}{l}\text { Déjudiciariser les } \\
\text { séparations : passage } \\
\text { non plus devant un } \\
\text { JAF, mais médiation } \\
\text { familiale }\end{array}$ \\
\hline LPLM & $\begin{array}{l}\text { Réflexion sur la } \\
\text { paternité /soutien } \\
\text { démarche }\end{array}$ & 2006 & $\begin{array}{l}\text { Associative } \\
\text { militante }\end{array}$ & $\begin{array}{l}\text { Accueil physique et } \\
\text { téléphonique }\end{array}$ & $\begin{array}{l}\text { Accompagnement } \\
\text { personnalisé sur les } \\
\text { dossiers ; pas de } \\
\text { réunions/ groupe de } \\
\text { parole }\end{array}$ & $\begin{array}{l}\text { Participation à des } \\
\text { comités de consulta- } \\
\text { tion }\end{array}$ & $\begin{array}{l}\text { Participer au débat } \\
\text { sur la parentalité } \\
\text { auprès des pouvoirs } \\
\text { publics }\end{array}$ \\
\hline Sos papa & $\begin{array}{l}\text { Égalité droit de } \\
\text { garde homme/ } \\
\text { femme post divorce } \\
\text { et soutien juridique }\end{array}$ & 1991 & $\begin{array}{l}\text { Associative } \\
\text { militante }\end{array}$ & $\begin{array}{l}\text { Réunions ; conseil } \\
\text { juridique sur le } \\
\text { dossier par les } \\
\text { animateurs }\end{array}$ & $\begin{array}{l}\text { Avocats ; Notaire ; } \\
\text { Psychologue }\end{array}$ & $\begin{array}{l}\text { Consultation et } \\
\text { lobbying }\end{array}$ & $\begin{array}{l}\text { Imposer notamment } \\
\text { la résidence alternée } \\
\text { par défaut }\end{array}$ \\
\hline Sos divorce & $\begin{array}{l}\text { Suppression presta- } \\
\text { tion compensatoire }\end{array}$ & 2000 & $\begin{array}{l}\text { Associative } \\
\text { militante }\end{array}$ & $\begin{array}{l}\text { Conseil personnalisé } \\
\text { dossier juridique }\end{array}$ & Liste d'avocats & & $\begin{array}{l}\text { Suppression de la } \\
\text { prestation compen- } \\
\text { satoire }\end{array}$ \\
\hline$A N C Q$ & $\begin{array}{l}\text { Suppression de la } \\
\text { pension alimentaire } \\
\text { pour conjoint sans } \\
\text { terme et promotion } \\
\text { de la garde partagée }\end{array}$ & 1999 & $\begin{array}{l}\text { Associative } \\
\text { militante }\end{array}$ & $\begin{array}{l}\text { Accueil } \\
\text { téléphonique }\end{array}$ & $\begin{array}{l}\text { Représentation } \\
\text { auprès des institu- } \\
\text { tions }\end{array}$ & & $\begin{array}{l}\text { C-422 : imposer la } \\
\text { garde partagée en } \\
\text { cas de conflit ; } \\
\text { suppression de la PA } \\
\text { sans terme pour le } \\
\text { conjoint }\end{array}$ \\
\hline $\begin{array}{l}\text { Après- } \\
\text { rupture }\end{array}$ & $\begin{array}{l}\text { Droit des pères après } \\
\text { la séparation }\end{array}$ & 1997 & $\begin{array}{l}\text { Associative } \\
\text { militante }\end{array}$ & $\begin{array}{l}\text { Accueil physique et } \\
\text { téléphonique }\end{array}$ & $\begin{array}{l}\text { Recherche (travail } \\
\text { statistique } \\
\text { notamment) }\end{array}$ & $\begin{array}{l}\text { Défense de droit / } \\
\text { accompagnement } \\
\text { des hommes ; arrêter } \\
\text { la propagande et le } \\
\text { « mensonge » } \\
\text { féministe }\end{array}$ & $\begin{array}{l}\text { Repenser les statisti- } \\
\text { ques sur les femmes } \\
\text { battues }\end{array}$ \\
\hline
\end{tabular}


Annexe 2 : suite

\begin{tabular}{|c|c|c|c|c|c|c|c|}
\hline Nom & But & $\begin{array}{l}\text { Date } \\
\text { création }\end{array}$ & Ressource & $\begin{array}{l}\text { Services } \\
\text { proposés }\end{array}$ & & & $\begin{array}{l}\text { Changements } \\
\text { législatifs proposés }\end{array}$ \\
\hline $\begin{array}{l}\text { F4J } \\
\text { Québec }\end{array}$ & $\begin{array}{l}\text { Droit des pères après } \\
\text { la séparation }\end{array}$ & 2004 & $\begin{array}{l}\text { Associative } \\
\text { militante }\end{array}$ & Conseil juridique & $\begin{array}{l}\text { Lobbying auprès des } \\
\text { institutions }\end{array}$ & & $\begin{array}{l}\text { C-422 : imposer la } \\
\text { garde partagée en } \\
\text { cas de conflit }\end{array}$ \\
\hline $\begin{array}{l}\text { Auton- } \\
\text { hommie }\end{array}$ & $\begin{array}{l}\text { Service social/ } \\
\text { Action individuelle } \\
\text { en ou groupe }\end{array}$ & 1982 & Institutionnelle & $\begin{array}{l}\text { Soutien psychologi- } \\
\text { que / Groupe de } \\
\text { parole }\end{array}$ & Conseil juridique & $\begin{array}{l}\text { Valorisation de } \\
\text { l'investissement } \\
\text { paternel }\end{array}$ & Aucun \\
\hline Repère & $\begin{array}{l}\text { Service social/ } \\
\text { Action individuelle } \\
\text { ou en groupe }\end{array}$ & 1995 & Institutionnelle & $\begin{array}{l}\text { Soutenir les pères } \\
\text { qui vivent des } \\
\text { situations difficiles } \\
\text { (séparations) }\end{array}$ & $\begin{array}{l}\text { Outiller les pères } \\
\text { dans le cadre des } \\
\text { développements des } \\
\text { compétences pater- } \\
\text { nelles qu'ils soient } \\
\text { en couple ou non. }\end{array}$ & & Aucun \\
\hline$R V P$ & $\begin{array}{l}\text { Valorisation de la } \\
\text { paternité auprès des } \\
\text { pouvoirs publics }\end{array}$ & 1997 & Institutionnelle & $\begin{array}{l}\text { Valorisation de la } \\
\text { paternité auprès de } \\
\text { la population en } \\
\text { général par des } \\
\text { activités }\end{array}$ & $\begin{array}{l}\text { Susciter un question- } \\
\text { nement sur les } \\
\text { services et actions } \\
\text { des organismes } \\
\text { quant à la place du } \\
\text { père dans leurs } \\
\text { interventions }\end{array}$ & $\begin{array}{l}\text { Soutenir/ regrouper } \\
\text { les organismes qui } \\
\text { veulent prendre des } \\
\text { initiatives pour tenir } \\
\text { compte des pères } \\
\text { dans leurs interven- } \\
\text { tions }\end{array}$ & Aucun \\
\hline Orpères & $\begin{array}{l}\text { Groupe de paroles } \\
\text { non mixtes de pères } \\
\text { séparés ou en } \\
\text { couples }\end{array}$ & 1998 & Institutionnelle & Soutien individualisé & Groupe de parole & & Aucun \\
\hline Oxygène & $\begin{array}{l}\text { Service social/ } \\
\text { hébergement } \\
\text { d'urgence de pères } \\
\text { ayant ou non la } \\
\text { garde des enfants }\end{array}$ & 1989 & Institutionnelle & $\begin{array}{l}\text { Logement père pour } \\
\text { accueillir ses enfants } \\
\text { en situation } \\
\text { d'urgence }\end{array}$ & & & Aucun \\
\hline $\begin{array}{l}\text { Père } \\
\text { séparés }\end{array}$ & $\begin{array}{l}\text { Service social/ } \\
\text { Action individuelle } \\
\text { ou en groupe }\end{array}$ & 1998 & Institutionnelle & $\begin{array}{l}\text { Groupe de parole } \\
\text { (coaching, soutien) }\end{array}$ & $\begin{array}{l}\text { Conseil juridique ; } \\
\text { médiation familiale ; } \\
\text { psychologue }\end{array}$ & & Aucun \\
\hline
\end{tabular}

